\title{
The relationship between spermine content of human milk during the first postnatal month and allergy in children
}

\author{
Olivier Peulen', Walthère Dewé2 ${ }^{2}$ Guy Dandrifosse ${ }^{1, \star}$, Isabelle Henrotay' \\ and Nadine Romain ${ }^{1}$ \\ 'Department of Biochemistry and General Physiology, Institute of Chemistry B6c, University of Liege, \\ B-4000 Liege (Sart-Tilman), Belgium: ${ }^{2}$ Department of Medical Informatics and Statistics, Institute of \\ Pathology, University of Liege, B-4000 Liege (Sart-Tilman), Belgium
}

Submitted 20 October 1997: Accepted 19 May 1998

\begin{abstract}
Design: Qualitative case study and mathematical model.

Setting: Belgium

Objectives: To evaluate the correlation between the polyamine mean concentration of the milk drunk during the first postnatal month and the appearance of allergy in children who drank this milk.

Results: A model that describes the dependence of the allergy appearance with the spermine mean concentration of milk drunk during the first postnatal month was established.

Conclusions: This model shows that $5.02 \mathrm{nmol} \mathrm{ml}^{-1}$ of spermine is a critical value to prevent the appearance of allergy.
\end{abstract}

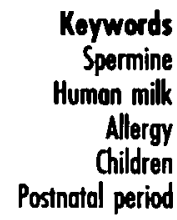

Polyamines (spermine, spermidine and putrescine) are small aliphatic polycationic molecules that are ubiquitous in nature and are synthesized by all cell types. These molecules are involved in intestinal growth ${ }^{1}$. In the rat, mucosal polyamine concentrations show a dramatic increase at the moment of postnatal intestinal maturation. Moreover, we have shown in previous studies that it is possible to induce precocious intestinal maturation in rats by giving them spermidine or spermine orally ${ }^{2}$. We have also previously shown ${ }^{3}$ that human milk contains generally more polyamines than infant formulas. Romain et $a l^{3}{ }^{3}$ proposed that the putative protective effect of human milk against allergies ${ }^{4}$, as compared to classical infant formulas, could at least partially be explained by its polyamine (spermine, spermidine, putrescine) concentration and the role of these compounds in the postnatal maturation of the small intestine.

Allergy is an important health problem as it restricts people suffering from it in their activities and social life. In this study we evaluated the correlation between the polyamine mean concentration of the milk drunk during the first postnatal month and the appearance of allergy in children who drank this milk.

\section{Material and methods}

\section{Selection of cases}

Five years after the birth of their child, a questionnaire was sent to nursing mothers, who had given their milk for polyamine concentration measurement in a previous study ${ }^{3}$. Of the 60 mothers who were asked 45 replied. The mothers provided information about the lactation duration and the eventual food or environmental allergy of their children, and this was either confirmed or invalidated by their GPs. The means of diagnosis (radioallergosorbent test, prick test, etc.) was decided by the GP. The diagnosis was blind to the polyamine status of the child

\section{Milk collection}

Preliminary studies were undertaken to determine the best moment of sampling during suckling ${ }^{3}$. The milk samples were collected in a standardized way according to Romain $e t$ al. ${ }^{3}$ Milk samples were also taken from the second breast just before the baby began to suckle it. Milk aliquots were frozen immediately after gathering and kept at $-20^{\circ} \mathrm{C}$ until analysis.

\section{Measurement of polyamine concentrations}

The polyamine concentrations were measured in the milk by using high-performance liquid chromatography (HPLC) $)^{3}$.

\section{Statistical analysis}

Difference between means was calculated by a nonparametric Mann-Whitney $U$ test. All other analyses were performed with the statistical software SAS. The probability of an allergy appearance was modelled as a function of spermine, spermidine and putrescine mean concentrations of milk secreted during the first 
postnatal month for each subject. To model the binary response $Y(Y=0$, not allergic; $Y=1$, allergic) we used the probability, $P(Y=1)$, and a logit transformation $\log [P /(1-P)]$ required in respect of the constraints for $P(0<P<1)$. The logit model is:

$$
\log \left(\frac{P}{1-P}\right)=a \times x+b
$$

where $x$ is the explanatory variable, and $a$ and $b$ the parameters. By isolating $P$ in the linear logistic model $\operatorname{logit}(P)=a x+b$, we get $P=\exp (a x+b) /$ $[1+\exp (a x+b)]$. A stepwise selection, an iterative procedure to select a model for a fixed $\alpha$ level, was used. Estimation of the parameters was made by maximum likelihood estimation using each subject separately. We modelled probability $(P)$ by using the logit of $P$ which was in fact the logarithm of the odds ratio. The critical point (cut off) of polyamine concentration below which there is a greater likelihood of becoming allergic with a maximum efficiency (specificity multiplied by sensitivity) was calculated by a receiver operating characteristic (ROC) analysis. Sensitivity and specificity were calculated for each concentration of spermine in the milk and used as critical points to classify each child as allergic or not. The best critical point is associated with the best efficiency.

\section{Results}

There was no apparent difference between the mothers who answered the questionnaire and those who did not regarding allergy or the polyamine content of their milk. In the mothers who did answer, $29 \%$ had an allergic child.

Mean concentrations of spermine/spermidine in milk drunk during the first postnatal month by allergic children and non-allergic children (Fig. 1) were statistically significantly different $(P=0.003$ and $P<0.001$ ).

It appeared that spermine mean concentration did predict allergy appearance. The model obeys the equation:

$$
P=\frac{e^{(2.506-0.689 \times x)}}{1+e^{(2.506-0.689 \times x)}}
$$

where $P$ is the probability of allergy appearance and $x$ is the spermine mean concentration during the first postnatal month. This provides statistical evidence of a relationship between the probability of developing allergy before 6 years of age and the spermine mean concentration $(P=0.0055)$ of the milk drunk during the first postnatal month (Fig. 2).

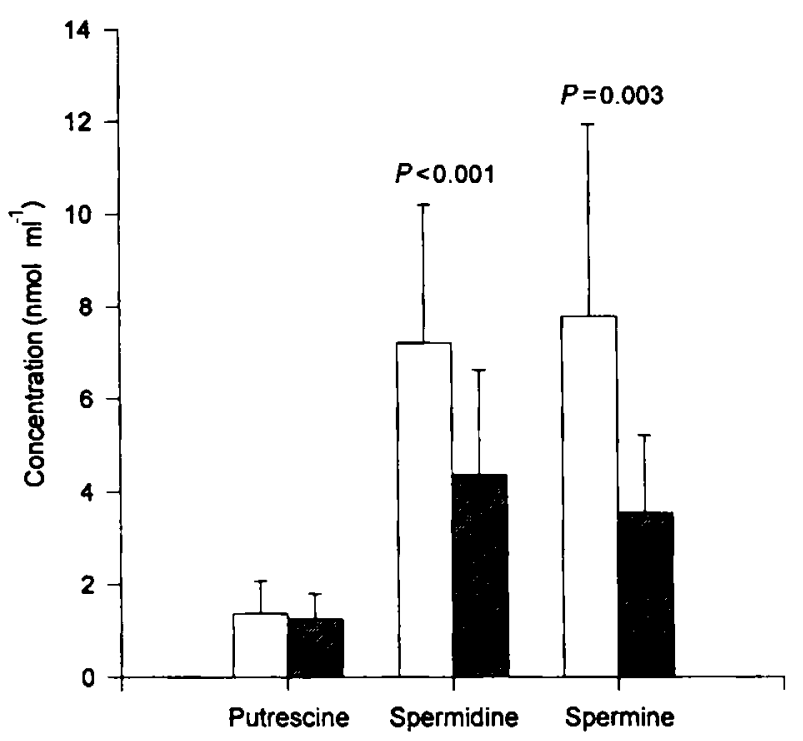

Fig. 1 Mean concentration of spermine, spermidine and putrescine (mean \pm SD) in milk drunk during the first postnatal month by allergic (hatched boxes) and non-allergic children (open boxes).

The proportion of correct predictions was $86 \%$ indicating an excellent fit of the model to the data. The comparison of the predicted by the logit model and the observed values for the variable allergy was $54 \%$ for specificity and $88 \%$ for sensitivity. This means that the probability that an allergic child was classified as allergic (true-positive) was 54\% and the probability that an nonallergic child was classified as non-allergic (truenegative) was $88 \%$ (Table 1 ).

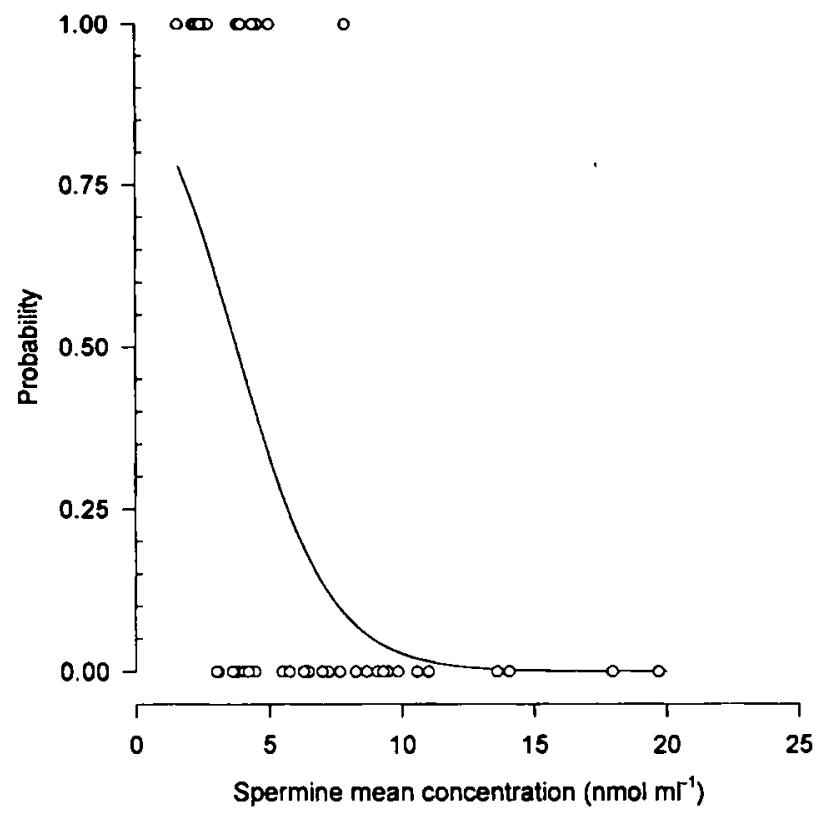

Fig. 2 Correlation between the spermine mean concentration in human milk secreted during the first postnatal month and the probability of allergy appearance in 5-year-old children. Circles are diagnosis of allergy ( $Y=0$, no allergy; $Y=1$, allergy) of each subject versus spermine mean concentration. The curve represents the probability derived from the logit model. 


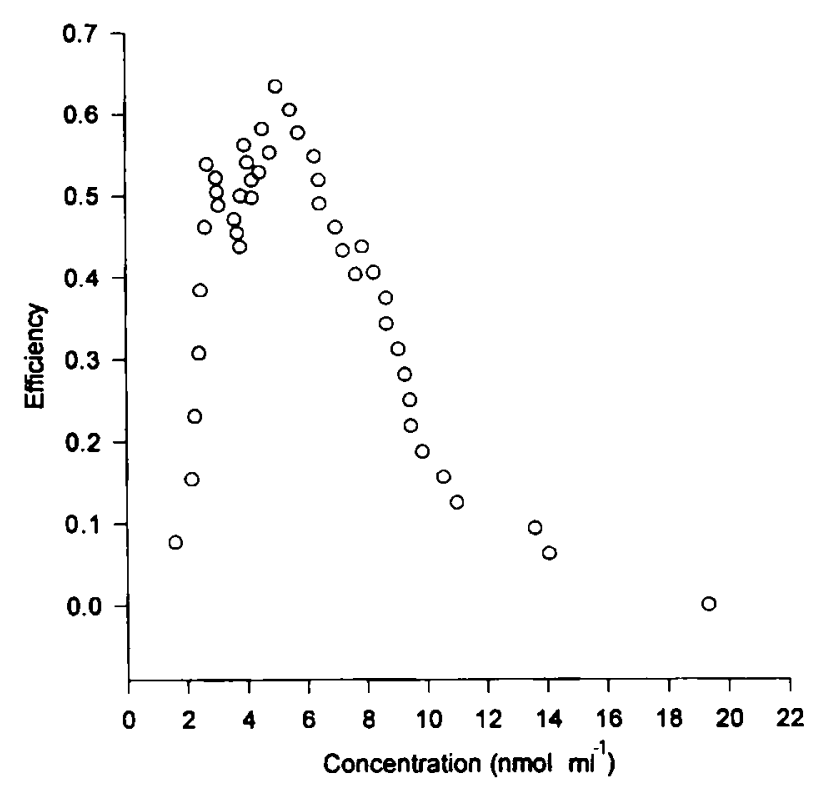

Fig. 3 Efficiency (sensitivity multiplied by specificity) determined by ROC analysis for each concentration of spermine as critical point versus spermine mean concentration $\left(\mathrm{nmol} \mathrm{ml}{ }^{-1}\right)$ in milk secreted during the first postnatal month.

Table 1 Specificity and sensitivity of the logit model

\begin{tabular}{lccc}
\hline & $\begin{array}{c}\text { Observed as } \\
\text { non-allergic }\end{array}$ & $\begin{array}{c}\text { Observed as } \\
\text { allergic }\end{array}$ & Total \\
\hline Predicted as non-allergic & $\underline{28}(88 \%)$ & 6 & 34 \\
Predicted as allergic & 4 & $\underline{7}(54 \%)$ & 11 \\
Total & 32 & 13 & 45 \\
\hline
\end{tabular}

Table 2 Specificity and sensitivity of the cut-off concentration of spermine

\begin{tabular}{lccc}
\hline & $\begin{array}{c}\text { Observed as } \\
\text { non-allergic }\end{array}$ & $\begin{array}{c}\text { Observed } \\
\text { allergic }\end{array}$ & Total \\
\hline Predicted as non-allergic & $\underline{22}(69 \%)$ & 1 & 23 \\
Predicted as allergic & 10 & $\underline{12}(92 \%)$ & 22 \\
Total & 32 & 13 & 45 \\
\hline
\end{tabular}

ROC analysis revealed that $5.02 \mathrm{nmol} \mathrm{m}^{-1}$ of spermine is the critical point above which a child has a greater likelihood of being non-allergic (Fig. 3). If the children were classified as allergic or non-allergic in accordance with this spermine mean concentration, the specificity and the sensitivity were 69 and $92 \%$ respectively (Table 2 ).

\section{Discussion}

Previous observations ${ }^{2,3.5}$ indicate that the spermine concentrations in milk drunk by babies could influence the passage of antigens from the intestinal lumen to the blood and thus the development of allergic reactions. Spermine seems to induce precocious postnatal maturation of the small intestine $e^{2}$ and the immune system $^{6}$. This spermine-induced maturation acts among other elements on disaccharidase-specific activities ${ }^{2}$, morphology and probably on gut closure. A study is currently being undertaken in the rat to determine what factors influence the level of polyamines in the milk. Preliminary results show that the composition of the food eaten by the mother can influence this parameter (Peulen et al., unpublished results).

During this study, we analysed the correlation between the spermine mean concentration in the milk drunk by babies during the first postnatal month and the appearance of allergy in these children later in life. By the method of sampling used ${ }^{3}$, the spermine concentration of the milk could not be proportional to the real intake of spermine in babies. However, a recent study indicates that this proportionality was respected ${ }^{7}$. Nevertheless we must bear in mind that this difference could be a potential bias in our study. Moreover, of 45 children we noted that $29 \%$ were allergic. This proportion is high in this population, indicating that our sample is not representative of the latter. Nevertheless, this final possibility cannot modify our conclusion based on the presence or absence of allergy in each child.

Our results indicate that the children who were allergic at the age of 5 years drank milk which contained less spermine and spermidine than the non-allergic children at the same age. This fact can be explained by increased postnatal maturation of the small intestine and the immune system. The increased maturation of the small intestine leads to gut closure which decreases the transfer of antigens from the lumen to the blood.

The logit model used shows that the development of allergy is dependent on the spermine mean concentration in the milk drunk during the first postnatal month. The sensitivity of this model was high $(88 \%$ true negative) but the specificity was lower ( $54 \%$ true positive) indicating that the model is better at finding the non-allergic than the allergic child. These results can be explained by a multifactorial origin of the allergy other than the weak spermine concentration of the milk. The spermine in the milk acts only as a preventive agent which regulates or induces small intestine maturation.

The critical point above which children have a greater likelihood of being non-allergic is $5.02 \mathrm{nmol} \mathrm{ml}^{-1}$ of spermine. If the children were classified as allergic or not according to this value of spermine mean concentration in their milk, it appears that specificity reaches 69\% (true-positive) and sensitivity 92\% (truenegative). Thus indicating that $5.02 \mathrm{nmol} \mathrm{ml}^{-1}$ of spermine could be used as an early diagnostic tool to determine if a child will develop allergy later in life. With this tool, $69 \%$ of the children who will become allergic could be detected early and treated with an hypoallergenic diet. Moreover, as spermine is not toxic at 
physiological doses, it could be added to milk formulas to prevent the development of the disease.

In conclusion, this study shows that allergy could be prevented in children by adapting the composition of the mother's milk. Moreover, children who are susceptible to allergy could be detected very early by an analysis of the mother's milk, and their diet or environment adapted to reduce the risk of disease. However, an extension of this study to a European level is necessary to provide further support to the conclusions of the present study.

\section{References}

1 Luk G. Polyamines in intestinal growth. Biochem. Soc. Trans. 1990; 18: 1990-1.
2 Dufour $\mathrm{C}$, Dandrifosse $\mathrm{G}$, Forget $\mathrm{P}$, Vermesse $\mathrm{F}$, Romain $\mathrm{N}$ Lepoint $A$. Spermine and spermidine induce intestinal maturation in the rat. Gastroenterolog $y$ 1988; 95: 112-16.

3 Romain $N$, Dandrifosse $G$, Jeusette $F$, Forget $P$. Polyamine concentration in rat milk and food, human milk and infant formulas. Pediatr. Res. 1992; 32: 58-63.

4 Iyngkaram N, Yadav M. Food allergy. In: March MN, ed Immunopathology of the Small Intestine. New York: John Wiley, 1987: 415-49.

5 Dandrifosse G. La spermine un agent préventif des allergies alimentaires? Arch. Int. Physiol. Biochim. 1993; 101 : A121.

6 ter Steege J, Buurman W, Forget P-P. Spermine induces maturation of the immature intestinal immune system in neonatal mice. J. Pediatr. Gastroenterol. Nutr. 1997; 25. 332-40.

7 Dorhout $\mathrm{B}$, van Beusekom CM, Huisman $\mathrm{M}$, Kingma AW, de $\operatorname{Hoog}$ E, Boersma ER, Muskiet FAJ. Estimation of 24-hour polyamine intake from mature human milk J. Pediatr. Gastroenterol. Nutr. 1996; 23: 298-302. 